\title{
NORMAL FORMS OF REAL SYMMETRIC SYSTEMS WITH MULTIPLICITY
}

\author{
P. J. BRAAM \\ The Mathematical Institute \\ 23-29 St. Giles, Oxford OX1 3LB, U.K. \\ E-mail:braam@maths.ox.ac.uk \\ J. J. DUISTERMAAT \\ Mathematical Institute, Utrecht University \\ P.O. Box 80.010, 3508 TA Utrecht, The Netherlands \\ E-mail:duis@math.ruu.nl
}

1. The result. I would like to start with formulating the result. In it, several concepts appear, with which the reader may be not so familiar. In the second part, these then will be discussed in more detail. The background material, and much more, can be found in [5].

Let $P$ be a (pseudo-)differential operator, acting on sections of an orthogonal vector bundle $E$ over an $n$-dimensional manifold $X$. All objects will be smooth, that is, infinitely differentiable. Because our results will be of a (micro-)local nature, we may use a local trivialization of $E$, in which $P$ is a square matrix of (pseudo-)differential operators. We will assume that in an orthogonal local trivialization the principal symbol $p(x, \xi)$ of $P$ is a real symmetric matrix; this is the case in many applications.

An important scalar function on the cotangent bundle $\mathrm{T}^{*} X$ of $X$ is

$$
\Delta(x, \xi):=\operatorname{det} p(x, \xi),
$$

the determinant of the principal symbol of $P$. Away from its zeros, $P$ behaves as an elliptic system. The set $N \subset \mathrm{T}^{*} X \backslash 0$ where $\Delta$ vanishes is called the

1991 Mathematics Subject Classification: 35G05, 35L40, 35L55, 35S05.

Lecture given by J. J. Duistermaat at the Banach Center Colloquium on 5th October 1993

The paper is in final form and no version of it will be published elsewhere. 
characteristic variety of $P$. At points of $N$ the polarization space $\operatorname{ker} p(x, \xi)$ has a positive dimension. At points $(x, \xi) \in N$ where $d \Delta(x, \xi) \neq 0$, the simple zeros of $\Delta, N$ is a smooth conical hypersurface in $\mathrm{T}^{*} X$.

If $\operatorname{dim} \operatorname{ker} p(x, \xi)=k$, then $\Delta$ has a zero of order $\geq k$ at $(x, \xi)$. Therefore, at the simple zeros of $\Delta$, the polarization space is one-dimensional and, modulo elliptic factors, the analysis can be reduced to considering a scalar operator with simple characteristics. For such operators the analysis modulo smoothing operators can be reduced to analysis of the operator $\partial / \partial x_{1}$, through conjugation with an elliptic Fourier integral operator, cf. [4, Sec. 6]. The translation of these results to the polarization properties of singular solutions to the original system has been carried out by Dencker [3].

For effects which are truly specific for systems we therefore must turn to the singular part $\Sigma$ of $N$, where $\Delta=0$ and $d \Delta=0$. For a generic symbol $\Sigma$ is stratified and the top stratum is a smooth codimension 3 submanifold of $\mathrm{T}^{*} X$, transversally to which $N$ looks like a quadratic cone. Modulo elliptic summands, $P$ can be reduced to a symmetric $2 \times 2$-system.

We now assume that $n \geq 3$. Our main result is that, by replacing the $2 \times 2$ operator $P$ by $A P A^{T}$, with $A$ a suitable elliptic Fourier integral operator, the full Taylor expansion of the principal symbol at $\Sigma$ can be brought into the very simple standard form

$$
p(x, \xi)=\left(\begin{array}{cc}
\mp \xi_{1} \pm \xi_{2} & x_{1} \xi_{3} \\
x_{1} \xi_{3} & \xi_{1}+\xi_{2}
\end{array}\right) .
$$

Note that in this case

$$
\Delta=\mp \xi_{1}^{2} \pm \xi_{2}^{2}-x_{1}^{2} \xi_{3}^{2}
$$

The two sign choices in (1.2) lead to drastically different behaviour of the solutions. For the plus sign, the operator is hyperbolic with respect to the variable $x_{1}$. Close to $\Sigma$, the bicharacteristic curves in the regular part of the characteristic set $N$ form helices, narrowly winding around smooth curves in $\Sigma$. Along with it, the polarization space rotates rapidly. For the minus sign, the operator is hyperbolic with respect to $x_{2}$. The bicharacteristic curves in $N$ approach $\Sigma$ and bounce away like a hyperbola approaching the intersection of its asymptotes. During the change of direction, the polarization space makes a quarter turn.

It should also be noted that this behaviour is very different from the so-called conical refraction, which only occurs in special cases, for instance if the medium is homogeneous (but non-isotropic).

This result appeared in Indagationes Mathematicae [2], in the volume on the occasion of the 70th birthday of Prof. Dr. J. Korevaar. In the paper, we also verify that the normal form (1.2) can be applied to Maxwell's equations and the equations for elastodynamics, for generic inhomogeneous media. The standard form (1.3) for $\Delta$ has been obtained before by Arnol'd, cf. [1, Sec. 8.1-8.4].

The normal form (1.2) for $P$ suggests that the solutions behave as if the system 
were equal to

$$
P=\frac{1}{i}\left(\begin{array}{cc}
\mp \frac{\partial}{\partial x_{1}} \pm \frac{\partial}{\partial x_{2}} & x_{1} \frac{\partial}{\partial x_{3}} \\
x_{1} \frac{\partial}{\partial x_{3}} & \frac{\partial}{\partial x_{1}}+\frac{\partial}{\partial x_{2}}
\end{array}\right) .
$$

Our goal is to understand properties of the solutions, such as propagation of the polarization of high-frequency solutions, by analyzing this model system. This information should complement the results on propagation of singularities obtained by [6] using energy estimates.

2. Background. We are concerned with systems of $k$ linear partial differential equations, of order $m$, for $k$ unknown functions. Equivalently, this can be written as an equation

$$
\left(P\left(x, \frac{1}{i} \frac{\partial}{\partial x}\right) u\right)(x)=0, \quad x \in \boldsymbol{R}^{n}
$$

in which $P$ is a $k \times k$-matrix of linear partial differential operators and the solution $u(x)$ is a vector-valued, $\boldsymbol{C}^{k}$-valued, function.

A plane wave with frequency vector $\xi \in \boldsymbol{R}^{n}$ and vector-valued amplitude function $a(x)$ is a function of the form

$$
u(x)=e^{i\langle x, \xi\rangle} a(x) .
$$

One can localize the investigation of $u(x)$ by multiplying it with a smooth cutoff function with support in the desired neighbourhood. The Fourier analysis says that every compactly supported distribution can be written as an integral over $\xi \in \boldsymbol{R}^{n}$ of plane waves. Singularities of the distribution come from the high frequency contributions, in the sense that the distribution is smooth if the Fourier coefficients $c(\xi)$ are rapidly decreasing as $|\xi| \rightarrow \infty$. That is, if for every $N$

$$
c(\xi)=\mathcal{O}\left(|\xi|^{-N}\right) \quad \text { as }|\xi| \rightarrow \infty \text {. }
$$

In this sense the investigation of singularities and of high frequency asymptotics are viewed as equivalent subjects.

One can localize the high frequency contributions also in $\xi$-space, by using cutoff functions with support in narrow cones. Or by replacing $\xi$ by $\tau \xi$, keeping $\xi$ in a small neighbourhood of a given nonzero $\xi_{0} \in \boldsymbol{R}^{n}$ and letting $\tau \rightarrow \infty$. The analysis of high-frequency waves with both $x$ and $\xi$ localized, and the corresponding analysis of singularities of distributions, is sometimes called micro-local analysis.

After a general substitution of variables, the linear phase function $x \mapsto \tau\langle x, \xi\rangle$ in the oscillatory factor will appear as the general real-valued function $\tau \varphi(x)$, at least if $\xi \neq 0$. Thus, in a coordinate-invariant setting, the high-frequency waves are of the form

$$
u(x)=e^{i \tau \varphi(x)} a(x),
$$

in which the phase function $\varphi(x)$ is a general real-valued smooth function of $x$. The frequency parameter $\tau$ is thought of as being large. The direction of the wave at $x$ is determined by the total derivative $d \varphi(x)$, which in the coordinate-invariant 
terminology is a linear form on the tangent space $\mathrm{T}_{x} X$ of the $x$-manifold $X$. That is, $d \varphi(x)$ belongs to the dual space $\left(\mathrm{T}_{x} X\right)^{*}$ of $\mathrm{T}_{x} X$. The $\mathrm{T}_{x} X$, together, for all $x \in X$, form the cotangent bundle $\mathrm{T}^{*} X$ of $X$. This is the phase space of classical mechanics, if the manifold $X$ is the configuration space $=$ the manifold of the positions.

The phenomenon which makes micro-local analysis work is that, asymptotically for high frequencies, linear partial differential operators act as multiplications by scalars, if we only look at the leading term of the asymptotic expansion. That is, there is a $k \times k$-matrix $p(x, \xi)$, depending smoothly on $(x, \xi) \in \mathrm{T}^{*} X$, such that

$$
P\left(e^{i t \varphi} a\right)(x)=\tau^{m} p(x, d \varphi(x)) e^{i \tau \varphi(x)} a(x)+\mathcal{O}\left(\tau^{m-1}\right), \quad \tau \rightarrow \infty,
$$

for any real-valued smooth phase function $\varphi(x)$ and $\boldsymbol{C}^{k}$-valued smooth amplitude function $a(x)$. The matrix-valued function $p(x, \xi)$ is uniquely determined by this property and is called the principal symbol of the operator $P$. For each $x \in X$, $\xi \mapsto p(x, \xi)$ is a homogeneous polynomial of degree $m$, hence in the asymptotics (2.5) we could equally well have inserted the frequency parameter $\tau$ in $p$ as a factor in front of $d \varphi(x)$. One says that (2.4) is an asymptotic solution of $P u=0$, if $P u$ is of smaller order than $\tau^{m}$ as $\tau \rightarrow \infty$.

The operator $P$ is called elliptic at $(x, \xi)$ if $p(x, \xi)$ is invertible. Clearly, $u(x)$ can only be an asymptotic solution if the amplitude vector $a(x)$ belongs to $\operatorname{ker} p(x, \xi)$, the polarization space of $P$ at $(x, \xi)$. Therefore, high-frequency solutions with nonzero amplitude vector can only occur if $(x, \xi)$ lies in

$$
N=\left\{(x, \xi) \in \mathrm{T}^{*} M ; \operatorname{det} p(x, \xi)=0\right\},
$$

the characteristic set of $P$.

We have now discussed almost all concepts mentioned in our normal form result, except for the pseudo-differential operators and Fourier integral operators.

In order to introduce pseudo-differential operators, we may start with the Fourier inversion formula, which states that

$$
u(x)=(2 \pi)^{-n} \int_{\boldsymbol{R}^{n}} \int_{\boldsymbol{R}^{n}} e^{i\langle x-y, \xi\rangle} u(y) d y d \xi
$$

valid for any temperate distribution in $\boldsymbol{R}^{n}$. Applying the linear partial differential operator $P$ and differentiating under the integral sign, we get

$$
(P u)(x)=(2 \pi)^{-n} \int_{\boldsymbol{R}^{n}} \int_{\boldsymbol{R}^{n}} e^{i\langle x-y, \xi\rangle} P(x, \xi) u(y) d y d \xi,
$$

in which the polynomial $\xi \mapsto P(x, \xi)$, called the total symbol of $P$ is obtained by formally substituting $i^{-1} \partial / \partial x_{j}$ by $\xi_{j}$ at every place. Clearly the principal symbol $p(x, \xi)$ is equal to the homogeneous part of degree $m$ of $P(x, \xi)$.

If one now replaces the polynomial $\xi \mapsto P(x, \xi)$ in (2.7) by a more general smooth function of $x$ and $\xi$, which has an asymptotic expansion as $|\xi| \rightarrow \infty$ in homogeneous terms $p_{m-j}(x, \xi)$ of decreasing order, then the operator $P$ is 
called a pseudo-differential operator of order $m$, with principal symbol equal to $p(x, \xi)=p_{m}(x, \xi)$. The assumptions are that for each integer $j \geq 0, p_{m-j}$ is a smooth function of $(x, \xi)$ for $\xi \neq 0$, which is homogeneous of degree $m-j$ :

$$
p_{m-j}(x, \tau \xi)=\tau^{m-j} p_{m-j}(x, \xi), \quad \tau>0 .
$$

The asymptotic assumptions are that for each $J \geq 1$ :

$$
P(x, \xi)-\sum_{j=0}^{J-1} p_{m-j}(x, \xi)=\mathcal{O}\left(|\xi|^{m-J}\right) \quad \text { as }|\xi| \rightarrow \infty,
$$

locally uniformly in $x$ and with similar estimates for all the derivatives. Such a function $P(x, \xi)$ is called a symbol of order $m$. Of course, in the case of systems, the symbols are taken to be matrix-valued. Like for differential operators, the pseudo-differential operator $P$ is called elliptic if the principal symbol $p(x, \xi)$ is invertible whenever $\xi \neq 0$.

Basic properties are that a pseudo-differential operator $P$ maps the Sobolev space $H^{s}(X)$ continuously to $H^{s-m}(X)$. Also, if $Q$ is a pseudo-differential operator of order $\mu$, then $P Q$ is a pseudo-differential operator of order $m+\mu$. Moreover, the principal symbol of $P Q$ is equal to the product of the principal symbols of $P$ and of $Q$, in this order. The last property follows from the fact that the asymptotic formula (2.5) also holds for arbitrary pseudo-differential operators $P$.

A basic application of the calculus of pseudo-differential operators is that if the pseudo-differential operator $P$ of order $m$ is elliptic, then it has a parametrix $Q$, which is a pseudo-differential operator of order $-m$, such that $P Q-I$ and $Q P-I$ are of order $-\infty$. One says: $P$ has a pseudo-differential inverse, modulo a smoothing operator. A well-known consequence is that every elliptic differential operator on a compact manifold $X$ is Fredholm. It therefore has a well-defined index

$$
\text { ind } P=\operatorname{dim} \operatorname{ker} P-\operatorname{dim} \text { coker } P \text {. }
$$

The next generalization consists of replacing the linear phase function

$$
(x, y) \mapsto\langle x-y, \xi\rangle
$$

in (2.7) by a more general real-valued smooth function $\psi(x, y, \theta)$. If $x$, resp. $y$, are points in a smooth manifold $X$, resp. $Y$, then $\psi$ is a smooth real-valued function on $X \times Y \times \boldsymbol{R}^{N}$, for some $N$. It is also assumed that the phase function $\psi$ is a homogeneous function of degree 1 of the auxiliary variable $\theta$, at least for sufficiently large $|\theta|$. That is,

$$
\psi(x, y, \tau \theta)=\tau \psi(x, y, \theta), \quad \tau \geq 1,|\theta| \geq M .
$$

An operator $A$ from functions on $Y$ to functions on $X$, given locally by integral formulas like

$$
(A u)(x)=\int e^{i \psi(x, y, \theta)} A(x, y, \theta) u(y) d y d \theta,
$$


in which $\psi$ is a phase function as just described and the amplitude $A$ satisfies the usual symbol estimates with $\xi$ replaced by $\theta$, is called a Fourier integral operator. Pseudo-differential operators are clearly a special case, with $X=Y$. Actually, for a coordinate-invariant definition of pseudo-differential operators on manifolds $X$, it is natural to allow more general phase functions, because linearity in $x$ does not make global sense in a general manifold.

For Fourier integral operators, an application of the method of stationary phase yields that $(2.5)$ now takes the form that $(P u)(x)$ is asymptotically given by $u(y)$, where the point

$$
(y, \eta)=(y, d \varphi(y)) \in \mathrm{T}^{*} Y
$$

is related to a point $(x, \xi) \in \mathrm{T}^{*} X$ by the condition that

$$
\begin{aligned}
\xi & =\frac{\partial \psi}{\partial x}(x, y, \theta) \\
\eta & =-\frac{\partial \psi}{\partial y}(x, y, \theta)
\end{aligned}
$$

for some $\theta$ such that

$$
\frac{\partial \psi}{\partial \theta}(x, y, \theta)=0
$$

The relation between $(y, \eta)$ and $(x, \xi)$ is not arbitrary: if it is a smooth mapping $\Psi$ from $\mathrm{T}^{*} Y$ to $\mathrm{T}^{*} X$, then it is a canonical transformation of the phase spaces, as defined in Hamiltonian mechanics. The homogeneity of $\psi$ also implies that it commutes with multiplication in the fibre by $\tau>0$, at least away from the zero section. An important fact is also that every homogeneous canonical transformation arises in this way from a suitable phase function $\psi$, so corresponds to a Fourier integral operator $A$.

A consequence of the calculus is the theorem of Egorov that if the Fourier integral operator is elliptic, and $P$ is a pseudo-differential operator in $Y$ with principal symbol equal to $p$, then $A P A^{-1}$ is a pseudo-differential operator in $X$ with principal symbol equal to $p \circ \Psi$. That is, a conjugation by means of elliptic Fourier integral operators can be used to transform a symbol by means of a substitution of variables given by an arbitrary homogeneous canonical transformation. In this way it becomes clear now that our normal form result is equivalent to the statement that the symbol can be brought into the standard form by a combination of the following operations:

(i) Left and right multiplication by suitable invertible matrix-valued symbols.

(ii) Substitution of phase space variables by means of a suitable homogeneous canonical transformation.

It is in the second step that symplectic geometry comes in. 


\section{References}

[1] V. I. Arnold, Singularities of Caustics and Wave Fronts, Mathematics and its Applications, Kluwer, 1990.

[2] P. J. Braam and J. J. Duistermaat, Normal forms of real symmetric systems with multiplicity, Indag. Math. N.S. 4 (1993), 407-421.

[3] N. Dencker, On the propagation of polarization sets for systems of real principal type, J. Funct. Anal. 46 (1982), 351-372.

[4] J. J. Duistermaat and L. Hörmander, Fourier integral operators. II, Acta Math. 128 (1972), 183-269.

[5] L. Hörmander, The Analysis of Linear Partial Differential Operators I-IV, SpringerVerlag, 1983-1985.

[6] V. Ya. Ivrii, Wave fronts of solutions of symmetric pseudodifferential systems, Siberian Math. J. 20 (1979), 390-405. 